\title{
Practical use of a word processor in a histopathology laboratory
}

\author{
JAMES C BRIGGS, NASSIF BN IBRAHIM, IAN MACKINTOSH, DAVID NORRIS \\ From the Departments of Histopathology and Physics, Frenchay Hospital, Bristol, BS16 1LE
}

SUMMARY Some of the facilities available with a commercially purchased word processing program, linked to a DEC PDP 11/23 computer are described, together with an account of the practical histopathological use. The system is based on a share of the computer with a Clinical Chemistry Department. Development was time-consuming and required the constant availability of the Department of Physics. However, once working, considerable saving in secretarial time has resulted and a number of projects have been started which would not have been contemplated without the use of the word processor and its linked computer.

Frenchay Hospital is situated in a semirural area on the north eastern outskirts of Bristol. It has regional and subregional specialist surgery (plastic, neuro and thoracic) as well as subserving the functions of a District General Hospital to the local community. The hospital is situated within half a mile of the M4 motorway between London and South Wales, and also within the same distance of the M32 running almost into the centre of Bristol. Because of the ease of transport into central Bristol, and the availability of jobs there, recruitment of secretarial staff at this hospital has not been easy and holiday periods have proved extremely difficult to cover. Additionally, over the past few years, the work load has increased so that the laboratory is now processing about 8500 surgical biopsies and about 500 post-mortems per year. This work load was too much for the existing secretarial staff (one full time and one part time), and with an almost inevitable growth occurring in the next few years, the concept of a word processor was explored.

TYPE OF EQUIPMENT AND ITS INSTALLATION Prior to the consideration of a word processor, the Clinical Chemistry Department had been pressing the Regional Health Authority for a computer. Eventually, through the foresight of the District Finance Officer, $£ 25000$ was made available from district finances for the purchase of both types of facility. At this time the hospital already had four other computers functioning in other departments. The machines come under the broad direction of the

Accepted for publication 2 July 1981
Department of Medical Physics. To capitalise on the expertise which already existed in the hospital it very rapidly became clear that the type of equipment to be purchased for the laboratory should be compatible with that already installed. In consequence, a Digital Equipment Corporation PDP11/23 was purchased with 256 kilobytes of memory and two 10 megabyte $15^{\prime \prime}$ rigid disk drives-that is, a disk storage capacity of what is equivalent to two Concise Oxford English Dictionaries. This contrasts with an 8" floppy disk which has a capacity of about 1 megabyte (about 200 dictionary pages).

Eight input/output devices can be attached to the computer; four of these are designated for clinical chemistry and four for histopathology. Of the latter four, one is a Diablo daisy wheel-type printer, housed in the histopathology office; a further two are visual display units (VDUs) with associated keyboards, for the two secretaries (Fig. 1). The fourth device is also a VDU, housed in one of three possible positions in the working area of the histopathology laboratory itself. The first of these positions is in the main processing and tissue-sectioning area; the second is in the cut-up room and the third in one of the consultants rooms. The systems have been operational and evolving since January 1981.

\section{FUNCTIONS OF A WORD PROCESSOR}

Very few laboratories in the United Kingdom appear to have word processing facilities at the moment. We feel that such equipment is likely to be evaluated by many pathology departments, particularly those facing a shortage in secretarial staff. A reasonably comprehensive description of what such facilities 


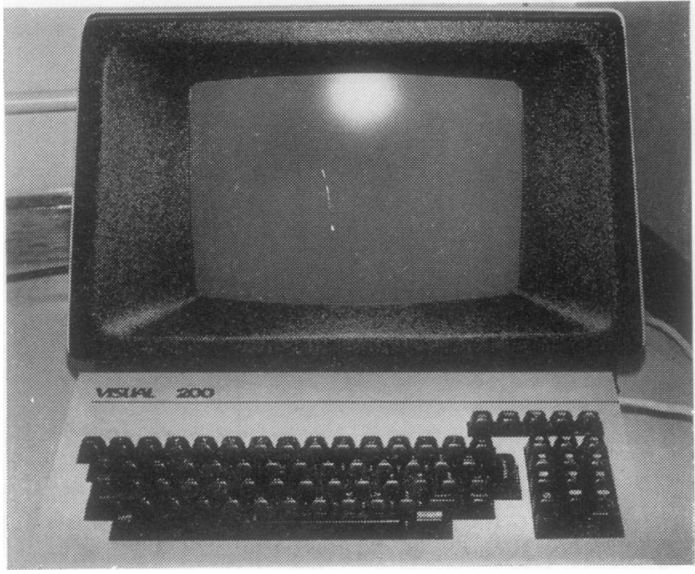

Fig. 1

offer is therefore likely to be of use to the vast majority of potential users.

Word processing consists of utilising the display facilities of a VDU attached to the computational power of a computer to create, manipulate and edit typed text. Therefore essentially any VDU/computer combination can be converted into a word processor if a suitable set of programs (software) is available. Although it is possible to write one's own word processing programs, given sufficient computer expertise and time, practicality dictates that the majority of people are likely to prefer to purchase an already established word processing system. The system eventually purchased for Frenchay was Lex 11 (Ace Micro-systems, London EC1.)

The general facilities available using the word processor package are covered by the "User Menu" (Fig. 2). The choice of an individual item on this

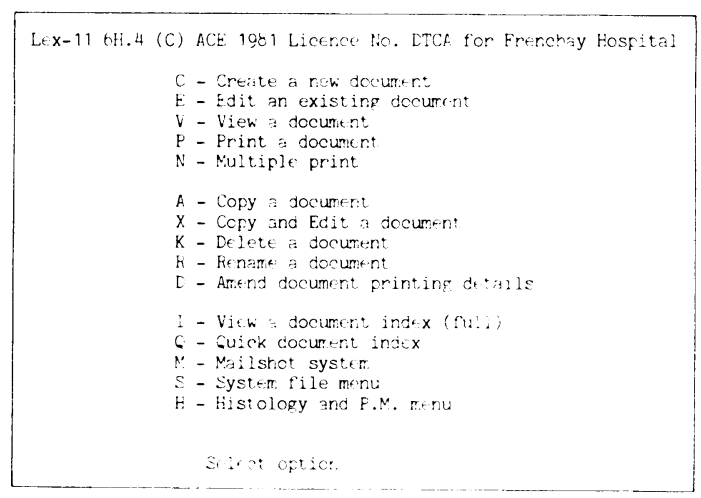

Fig. 2 menu is made possible by the code letters preceding each line. For instance, by depressing the character "I" on the VDU's typewriter-style keyboard, it is possible to view the Index File of all the documents stored in the computer. Having obtained the computer coded title for a particular document-for example, $9981 \mathrm{PM}$, it is possible either to view this document by depression of the " $V$ " key or to edit the same document by depressing the " $E$ " character. In order to condense the histological and postmortem requirements into a slightly simpler format, a special item " $\mathrm{H}$ " has been incorporated into the User Menu. This call up a "sub-menu" as illustrated in Figure 3.

Histology and P.M. Menu

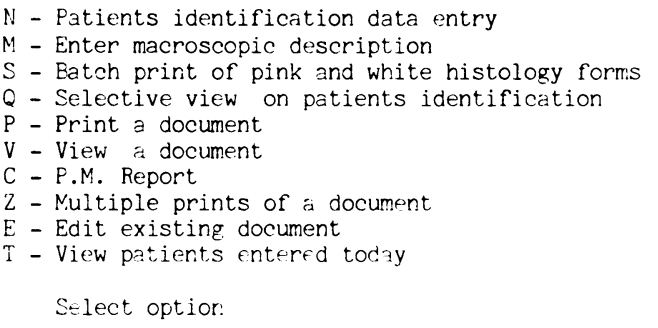

Fig. 3

Within the main User Menu an extremely important subsection is the System File Menu (character " $S$ "). This menu is illustrated in Fig. 4 and the subsection 1 is the most used. In this particular mode, it is possible to create large portions of often repeated text, to be stored in the computer in such a

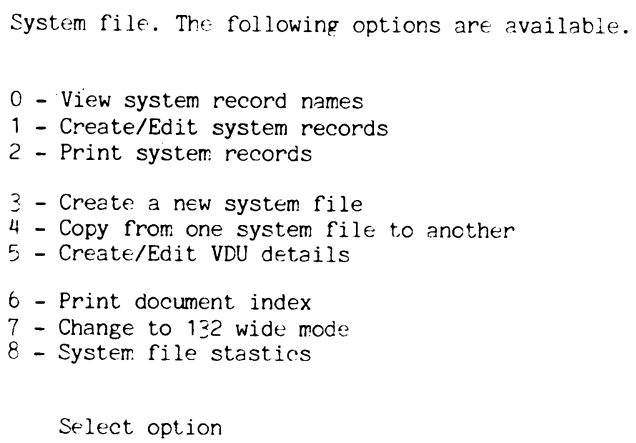

Fig. 4 
way that they are capable of being recalled at the touch of two or three buttons during the ordinary daily use of the machine.

Another important section of the user menu is called up by depressing character " $D$ ". This allows the skeletal format of new documents to be produced. (Figs. 5 and 6). Without the production of these skeletons the printer does not know where to start and stop on each sheet of paper. A particularly useful facility here is the ability to call up abbreviations. Two possibilities exist:

(a) One can type abbreviations, up to a maximum of 72 characters, onto the actual format details as displayed on the VDU. These abbreviations can then be recalled on a depression of only two buttons.

(b) A much more useful form of utilising this facility is to combine it with the previously mentioned System File index. Large portions of text, entered into the System File can be stored as abbreviations. Provided that the amount of text occupies no more than a "screenful" on the VDU, then the text is capable of being stored under a single abbreviation by the use of only four charac- ters. If more than a screenful of text requires to be recalled, then a further four characters under the same abbreviation heading is capable of recalling another screenful at the depression of the same two keys required to call up the first part of the abbreviation. This is perhaps best understood by reference to Figures 6 and 7.

From this it can be seen that the entire postmortem document is stored under abbreviation 1 , in two portions. The first of these is $\langle\mathbf{P M}\rangle$; the second of these is $<C O M>$. Each of the angled brackets calls up a screenful of information stored in the System File. Such a screenful is illustrated in Figure 7.

From the format in Fig. 7 it can be seen that a considerable amount of information is stored in a horizontal plane. For practical purposes this text needs to be expanded and displayed in a somewhat different manner when being used for day-to-day typing. The useful format of the information seen in Fig. 7 is shown in Figure 8.

Although our word processing system came with an instruction manual, the assistance of graduate

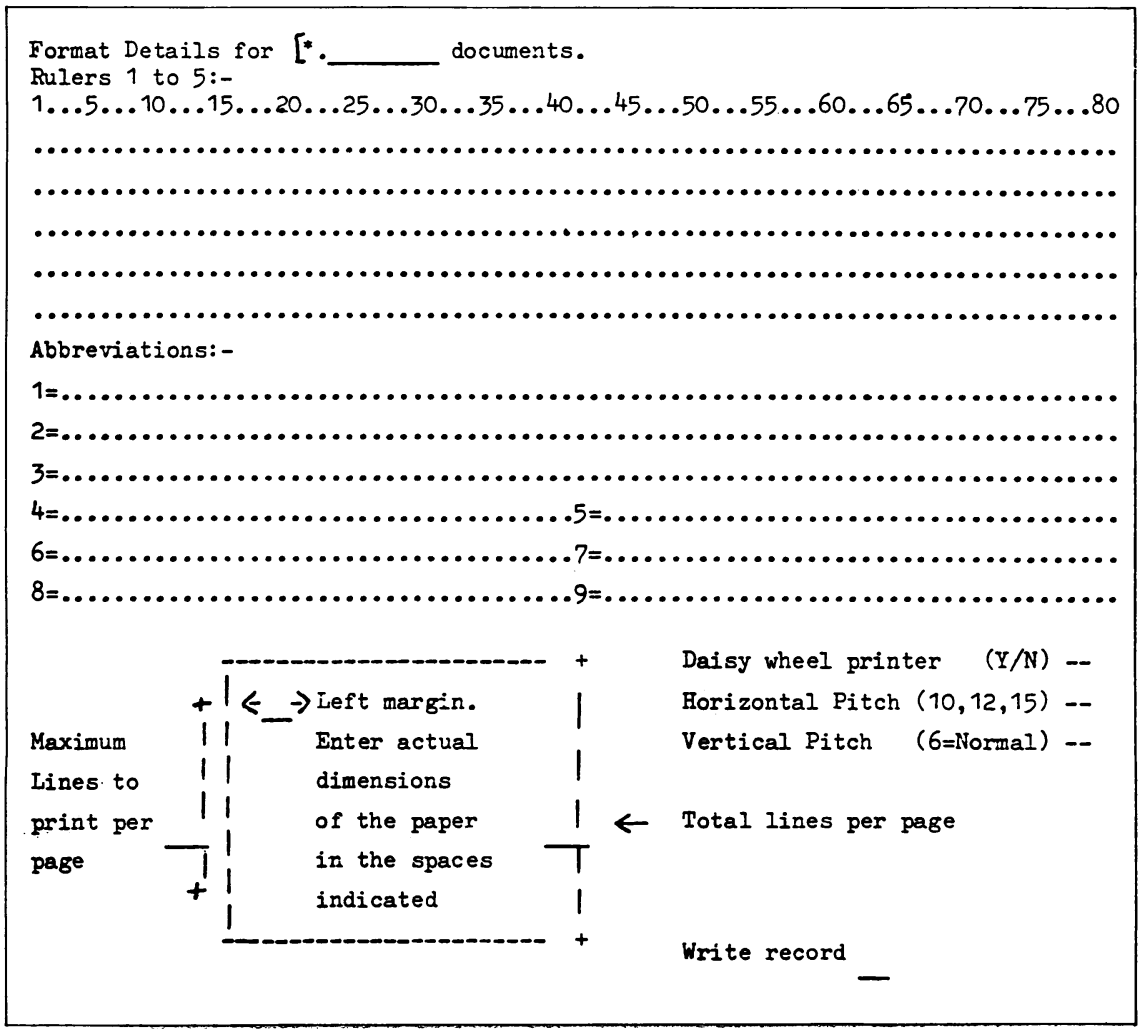

$2^{*}$
Fig. 5 Basic document format details. The rulers govern the number of characters per line. The abbreviations can be used to recall up to nine separate pieces of stored text of varying length. 


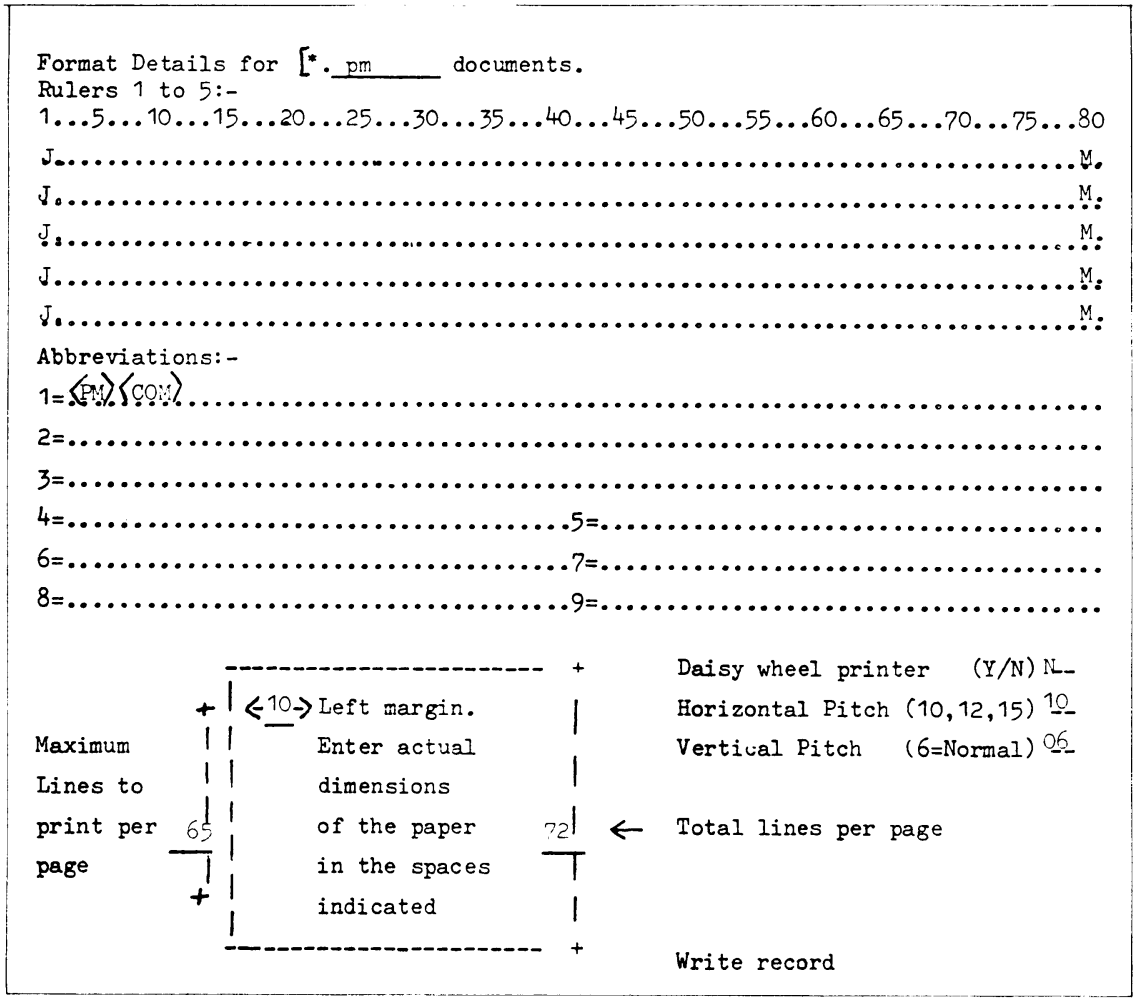

Fig. 6 Post-mortem document format details. The " $J$. ....M" ruler section causes the final printed version to have justified margins. The angled bracket symbols under. abbreviation 1 indicate two separate system file entries stored as this abbreviation.

physicists in applying these instructions and sorting out problems proved invaluable, and in some instances mandatory.

\section{PRACTICAL USAGE}

Post-mortem reports

The basic post-mortem format, programmed as above, provides the framework for each postmortem report. Into the program have been intro- duced a number of pauses for the insertion of patients personal information, date and time of examination etc. The first major pause in the format then comes under HISTORY. Any amount can be typed into this particular blank. The program automatically adjusts the space available to fill exactly the amount of text required. The next major pause is for EXTERNAL EXAMINATION, where similar remarks apply. Under the general heading INTERNAL

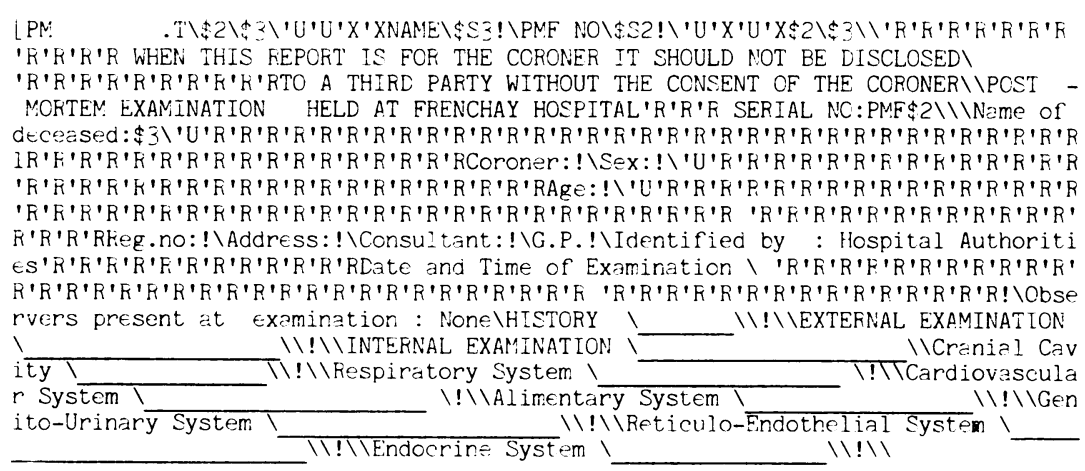

Fig. 7 The format of the first part of the post-mortem document as stored in the system file. (The exclamation marks (!) are replaced by question marks (?) in the used version; see Fig. 8). 
W'HEN THIS REPORT IS FOR THE CORONER IT SHCULD NOT BE DISCLOSED

TO A THIRD PARTY WITHOUT THE CONSENT OF THE CORCNER

PCST -MORTEM EXAMINATION HELD AT FRENCHAY HOSPITAL SERIAL NO: PMF ??

Name of

deceased:??

Coroner:??

Sex:??

Age:?? Reg.no:??

Consultant:??

G.P.??

Identified by : Hospital Authorities

Date and Time of Exarrination ??

Observers present at examination : None

HISTORY

??

EXTERINAL EXAMINATION

??

INTERNAL EXANINATION

Cranial Cavity

??

Respiratory System

??

Cardiovascular System

??

Alimentar'y Systemi

Nouth normal. Tongue normal. Oesophagus normal. Peritoneum normal. Stomach internally and externally normal. Duodenum internally and externally normal. The remainder of the intestines was externally rormal. The liver weighec ?? grams and was unremarkable both externally and on cut section. The gali bladder and all associated ducts were normal. The pancreas was normal.

Genito-Urinary System

??

Reticulo-Endothelial System

??

Endocrine System

??

Fig. 8 Part of the basic post-mortem document. The "Alimentary System" has been filled in with the memory-stored description, which allows major or minor editing. The question marks (??) indicate where specific pauses have been built into the system, but it is possible to edit any part of the text.

EXAMINATION a number of major systems have been incorporated. Each of these has an appropriate blank space governed by a pause in the program. Within this particular part of the post-mortem record, it has been found that one or more systems are frequently virtually within normal limits. Because of this observation, it has proved possible to insert, via the System File Menu, a whole series of preprogrammed features, each punctuated with appropriate pauses to allow the insertion of organ weights, and each capable of being edited to a greater or lesser extent to record the precise findings under the given system. Figure 8 shows the text of the standard "Alimentary System" finding.

\section{Histological reporting}

Specimens are received from the Theatres and personal identification data is entered using the appropriate section on the Histology submenu (see Fig. 3). This information is stored in the computer memory. At the time of the cut-up of the surgical specimens the macroscopic appearances are dictated onto tape. There are a number of specimens likely to have a relatively standard macroscopic description; some of these will also probably have a standard histological appearance. Examples of standard macroscopic descriptions, some of which are associated with standard histological appearance, have been stored in the computer memory, using the System File facilities. One laboratory in the United States $^{1}$ has at least 150 disk-stored gross and microscopic description formats that are available for instant recall. In our laboratory, a VDU is available in the cut-up room. When the pathologist performing the cut-up encounters a specimen which is likely to conform to one of the standard descriptions, this description can be called up almost instantaneously on the VDU screen to be checked. Like the post-mortem format described above, all histological material has pauses programmed into 
the text to enable the insertion of sizes and/or weights at appropriate points. Should a specimen conform, or nearly conform, to one of the standard stored texts the pathologist performing the cut-up can indicate to the secretary that she should call up this standard text, which requires only the depression of three or four keys, and insert the appropriate organ sizes, and other modifications, as dictated by the pathologist.

When the cut-up is complete, the tape, together with the request forms, which include the appropriate laboratory reference number, go for typing. The secretary calls up the previously entered personal details of the patient by typing the laboratory reference number. She then adds either the dictated macroscopic description or calls up from the computer memory a preprogrammed description and inserts the appropriate modifications as indicated. These reports therefore consist of the preliminary production phases of the final macroscopic and histological opinion which will eventually be incorporated into the patients' notes. Some of these, as indicated earlier, may well have, in their preliminary form, a guessed histological opinion. The reports are subsequently batch-printed, on yellow paper, at a convenient time during the day. This batch-printing utilises a special hopper head on the printer which feeds single sheets into the machine. Generally 30-40 reports are generated at a time and théy are produced in numerical order, irrespective of the order in which they were entered into the computer.

The histological slides, together with the preliminary reports (printed on yellow paper), eventually come to the pathologist responsible for the cut-up. He checks the macroscopic description and dictates modifications as necessary. He then adds the histological diagnosis. In those instances where the histological diagnosis has been guessed at the time of cut-up, this opinion can be confirmed or refuted. If confirmed, this is indicated to the secretary; if refuted a new histological diagnosis is dictated. The secretary enters all the modifications into the computer and subsequently prints the final reports, this time on pink paper for the wards and white paper for the laboratory records. Again batch printing is utilised, but with the added refinement of sub-batching each pathologist's reports; this saves later sorting. The use of pink forms is a security measure against the possibility of one of the yellow preliminary reports, which may incorporate a histological opinion, from being despatched to the wards. Further precautions to prevent this type of error have also been incorporated, the most important of which is that the preliminary forms contain no provision for a pathologist's signature.

Of fundamental importance in any histological laboratory is the retrieval of data. Any of the patient's personal details, as originally entered at the time of receipt of the specimen, can be used to call up information. For instance, all the biopsies for a given surname can be rapidly recalled; a specific biopsy, by means of an individual laboratory biopsy number, can also be rapidly recalled. Copies of this information are easily printed for despatch to the patient's notes. Permanent laboratory records of this information has been maintained initially by keeping the traditional copy in laboratory files. As confidence in the system grows, it is expected that this paper hard copy will be gradually phased out, until the entire record system is maintained within the computer.

Long term retrieval, particularly for research purposes, entails the use of filing systems of some complexity. At the moment the current filing system used in this laboratory is relatively simple and only enables easy access to broad disease groups - for example, carcinomas of lung; it does not allow easy access to various subgroups - for example, oat cell carcinomas. With the advent of the word processor, we have decided to utilise the SNOP classification system. ${ }^{2}$ Manually this is time-consuming, much more so than our current filing system. Additionally, SNOP coding manuals would have to find room on already overcrowded laboratory benches! These problems have been overcome by a program, developed in the Department of Medical Physics, whereby a series of key words are added to the bottom of each report. These key words relate to a SNOP dictionary and the computer automatically gives a correct SNOP coding, as indicated by the key words. The key words themselves do not appear on the report. Automatic SNOP coding systems have already been published. ${ }^{34}$ An automatic system, based on a word processor and utilising the Northwick Park program, is in use at the Leicester General Hospital (EH McKay, personal communication, 1981).

\section{Mailshot potential}

This utilises the facility of the machine to take a standard letter and to send it, individually named and addressed to any person who happens to be on the mailing register for that particular type of letter. This facility has been used in the formation of a National Melanoma Histology Group. This is in its early stages but already a number of histopathologists interested in this subject are on the computer's mailing list. Regular correspondence, including annotation of recent literature, is easily dispatched. Specific future uses of this facility revolve, initially, 
around the malignant melanomas treated in this hospital. At the moment a melanoma register exists of all patients seen and treated. Follow-up from their GP has proved administratively difficult. With the use of the mailshot facility regular followup letters to the GPs will be sent at six monthly intervals with the minimum of secretarial effort.

\section{Writing papers}

The possession of a word processor makes the writing, if not the actual production, of scientific papers very much easier. Rough drafts can be entered into the computer memory and edited with ease. It is not uncommon to find a large percentage of the original draft text to be usable in the final paper; the computer makes the use of this original text extremely easy. The quality of the final typed production is of high standard and, using some of the other word processing facilities, it is possible to title individually and number each page of text, quite automatically. Sorting and storage of some of the literature may be an additional possibility.

\section{Security}

It is of paramount importance, when large amounts of information are stored on a computer disk, that some steps are made to prevent loss of this information should a computer break down, or similar tragedy, occur. This is overcome by the availability of a duplicate rigid disk, which is up-dated each morning and stored separately from the computer itself.

\section{Cytology}

This laboratory has only a small cytology load; in consequence no provision has been made for the separation of this material from the routine histology. Asworth et al. ${ }^{5}$ have published information on this subject using their word processor computer.

\section{Relation to chemistry}

As indicated, half the computer facilities are used by Clinical Chemistry. About $20 \%$ of the routine work load is managed by the computer at present; automated work has yet to be integrated. There has been no clash of interests between the two users and the only observed effect is a slow down in the processing of material when both departments are using their facilities fully. This effect was, on occasion, quite frustrating. The Department of Physics produced new programs and the situation is now much improved.

\section{Advantages}

There has been a marked saving in the time taken to prepare post-mortem reports. An estimated
$50-60 \%$ reduction is typing time has resulted. Additionally the quality of the printed version is now uniformly excellent and multiple copies are produced with ease. The time spent actually dicating the post-mortem findings has been reduced by 10 $60 \%$, depending on the case, with the ability to inform the typist that certain standard phrases, with or without editing, can be inserted. Histologically the saving in time is much less marked. Although some programmed phrases can be used, this is offset by the introduction of the SNOP coding system which requires keywords typed to the bottom of each report. However, without the computer it is most unlikely that this laboratory would have adopted SNOP or any other similar system.

\section{Disadvantages}

After initial teething problems, some of which were major, there have proved to be very few disadvantages. The secretarial staff learned to use the basic word processing facilities rapidly. Use of some of the more sophisticated aspects took longer, and some of the detailed programming has been left to the medical or physics staff. Printing of documents requires a degree of organisation not previously necessary-for example, the batch printing must be completed in time to catch the twice daily delivery of reports to the wards. As a result there has been some loss of flexibility. One small disadvantage is that the machine is a profligate user of paper and printer ribbon. None of the printed paper can be used twice or, conveniently on both sides.

Development of the system was time-consuming and required the constant availability of the Department of Physics. Laboratories without ease of access to such a Department may well find the introduction of a word processor not worth while, except on a simple level.

\section{References}

${ }^{1}$ Ashworth CT, McConnell TH, Nielsen CR, Ashworth RD, Sandford J. A computerised word-processing and data system for histology in a private medical laboratory. Am J Clin Pathol 1979;71:257-62.

${ }^{2}$ College of American Pathologists. Systemised nomenclature of pathology. Chicago, Ill: College of American Pathologists, 1969.

${ }^{3}$ Coles EC, Slavin G. An evaluation of automatic coding of surgical pathology reports. J Clin Pathol 1976;29:621-5.

4 Foulis PR, Norbutt AM, Mendelow H, Kessler GF. Pathology accessioning and retrieval system with encoding by computer (PARSEC). A microcomputerbased system for anatomic pathology featuring automated SNOP coding and multiple administrative functions. Am J Clin Pathol 1980;73:748-53.

5 Ashworth CT, McConnell TH, Nielsen CR, Ashworth RD, Sandford J. A computer system for cytology in a private laboratory using a PDP 8/E minicomputer. $\mathrm{Am} \mathrm{J} \mathrm{Clin}$ Pathol 1979;71:412-7. 
Requests for reprints to: Dr JC Briggs, Department of Histopathology, Frenchay Hospital, Bristol BS16 1LE, England.
Further reading: Med Lab Sci 1980;37:193-222. (A series of articles on computers in medical laboratories.) Microprocessors. A short introduction. 1981. Department of Industry, Dean Bradley House, 52 Horseferry Road, London SW1 2BR. 